\title{
NOTES
}

\section{A jiffy clock for the Apple IIc, II + , and IIe with a mouse}

\author{
CHARLES MERBITZ, JEFFREY GRIP, \\ and HANS PETER MARQUI \\ Rehabilitation Institute of Chicago, Chicago, Illinois
}

A "jiffy clock" is presented for the Apple IIc or other Apple II having Apple's mouse card. The clock allows timing of events to tenths of a second, and is read from BASIC. A convenient elapsed timer function is provided as well.

Unlike with some microcomputers (e.g., Radio Shack TRS-80; Balsam, Deich, O'Connor, \& Scopatz, 1986) that provide a current time function, timing events with the earlier Apple II, II+, IIe, and Ilc usually requires the addition of a hardware clock (Flowers, 1982). A clock has been built into the new Apple IIgs; with the Apple IIc, a $60-\mathrm{Hz}$ interrupt is readily available and can be used to run a simple "jiffy clock." Since the jiffy clock originates in Apple's mouse firmware, an Apple II+ or Ile with a mouse card has access to the jiffy clock.

The routines described here create a jiffy clock that keeps time to tenths of a sec and is suitable for general use. They require Applesoft floating-point BASIC. Because the clock pauses during disk access, its accuracy is compromised when disk operations take place during timing.

ProDOS was designed to handle interrupts; DOS 3.3 was not. Therefore, different versions of the jiffy clock program are provided. For the DOS 3.3 version, it is strongly suggested that the clock be turned off with a CALL 777 before issuing any DOS commands, since both DOS and the clock use location $\$ 45$ (decimal 69). This precaution is not needed in the ProDOS environment, or with an enhanced IIe or IIl.

To type in the program listing, one must boot the preferred DOS and enter the monitor with a CALL-151 $<$ RETURN $>$. Each line of the program may be entered as follows. The four-digit hexadecimal address (e.g., 02BD in DOS 3.3, or 02B5 in ProDOS), a colon, and the address contents are entered, and <RETURN $>$ is pressed. No space occurs between the colon and the first

This work was supported by National Institute of Handicapped Research Grant G008300079. The authors also wish to recognize the constructive suggestions of the reviewers. Address correspondence to Charles Merbitz, Rehabilitation Institute of Chicago, 448 E. Ontario, 6th Floor, Chicago, IL 60611 . pair of numbers, but a space separates each subsequent pair. The remainder of the program can be entered in the same manner. To return to BASIC when finished, type $<$ Control-C $><$ RETURN $>$.

To save the clock to disk with DOS 3.3, BSAVE JFFY, A\$2BD, L223 should be entered. With ProDOS, BSAVE JIFFY, A\$2B5, L245 will save the clock to disk. The clock may be installed either before a BASIC program runs or within a BASIC program. The command BLOAD JIFFY retrieves the clock from the disk.

The memory locations used to control the clock are the same in both versions. POKE is used to set the time base to seconds (POKE 786,60) or tenths of seconds (POKE 786,6 ). Because the time is stored in 2 bytes, at 65,536 $(64 \mathrm{~K})$ increments (either seconds or tenths of seconds), the clock reaches its maximum value and "rolls over" to zero before continuing to increment. The possibility that a session will last long enough to include a rollover must be considered when programming. Following a rollover, a negative number will result when the elapsed time is calculated. (Expansion to 4 bytes to increase the duration before a rollover is possible, but does not seem useful in view of the interruption-during-diskaccess problem.) Execution of a CALL 768 locates the slot of the mouse card, and a CALL 771 begins the clock. The clock can be disabled with a CALL 777.

From within a BASIC program, execution of the command \& $A$ in any line reads the clock into the variable $A$. Any legal floating-point variable can be used, but not a string, integer, or array variable. A CALL 774 clears the clock to zero but does not stop it. The next \& A will then give the time since the most recent CALL 774. This feature is convenient when the clock is used repeatedly as an elapsed timer. Otherwise, the programmer must store the first time value when timing begins and subtract it from the second time value when timing ends to find the elapsed time.

Availability. The jiffy clock is available on disk to persons who send $\$ 4$ or a labeled, initialized 5.25-in. diskette with a self-addressed, stamped mailer to the senior author. Please specify which DOS is preferred, or send a double-sided, double-notched diskette for both versions.

\section{REFERENCES}

Balsam, P., Deich, J., O'Connor, K. \& Scopatz, R. (1986). Microcomputers and conditioning research. Behavior Research Methods, Instruments, \& Computers, 17, 537-545.

Flowers, J. H. (1982). Some simple Apple II software for the collection and analysis of observational data. Behavior Research Methods \& Instrumentation, 14, 241-249.

(Revision accepted for publication November 24, 1987.) 